\title{
On the Sharp Triangle Inequalities in Quasi-Banach Spaces
}

\author{
Xiangzhen Xiao \\ Experimental Center of Henan Institute of Science and Technology \\ Xinxiang, Henan, P. R. of China \\ Email: xiaoxxz@163.com
}

\begin{abstract}
The triangle inequality is one of the most important and fundamental inequalities in analysis. Many authors have been treating its generalizations and reverse inequalities. In this paper, we shall present the sharp triangle inequality and its reverse inequality for an arbitrary number of finitely many nonzero elements of a quasi-Banach space, which generalize the results obtained by $\mathrm{C}$. $\mathrm{Wu}$ and $\mathrm{Y}$. $\mathrm{J}$. $\mathrm{Li}$ in [1].
\end{abstract}

Keywords-Triangle inequality; Sharp triangle inequality; Reverse inequality; Norm; Quasi-Banach spaces

\section{INTRODUCTION}

The triangle inequality is one of the most fundamental inequalities in analysis and has been treated by many authors (see [2], [3], [4], [6] among others). Recently C. $\mathrm{Wu}$ and Y. J. Li [1] showed the following sharp triangle inequality and its reverse inequality in a quasi-Banach space.

Theorem I.1. For all nonzero elements $x, y$ in a quasiBanach space $\mathrm{X}$ with $\|x\| \geq\|y\|$, then

$$
\begin{aligned}
\|x+y\| & +C\left(2-\left\|\frac{x}{\|x\|}+\frac{y}{\|y\|}\right\|\right)\|y\| \\
& \leq C(\|x\|+\|y\|) \\
& \leq\|x+y\|+\left(2 C^{2}-\left\|\frac{x}{\|x\|}+\frac{y}{\|y\|}\right\|\right)\|x\|,
\end{aligned}
$$

where $C$ is a constant and $C \geq 1$.

Let us recall some basic facts concerning the quasiBanach spaces and some preliminary results(see [7] for more information about the quasi-Banach spaces).

Definition I.2. (see [7]) Let $X$ be a linear space. A quasi-norm is a real-valued function on $\mathrm{X}$ satisfying the following:

(1) $\|x\| \geq 0$ for all $x \in X$ and $\|x\|=0$ if and only if $x=0$;

(2) $\|\lambda x\|=|\lambda| \| x||$ for all $\lambda \in \mathbb{R}$ and all $x \in X$;

(3) There is a constant $K \geq 1$ such that such that $\|x+y\| \leq \mathrm{K}(\|x+y\|)$ for all $x, y \in X$.

The pair $(X,\|\cdot\|)$ is called a quasi-normed space if $\|\cdot\|$ is a quasi-norm on $\mathrm{X}$.

A quasi-Banach space is a complete quasi-normed space.
A quasi-norm $\|\cdot\|$ is called a $\mathrm{p}-\operatorname{norm}(0<\mathrm{p} \leqslant 1)$ if

$$
\|x+y\|^{p} \leq\|x\|^{p}+\|y\|^{p}
$$

for all $x, y \in X$. In this case, a quasi-Banach space is called a $\mathrm{p}$-Banach space.

In this paper, we shall generalize the inequalities (1) and (2) for an arbitrary number of finitely many nonzero elements of a quasi-Banach space.

\section{M AIN RESULTS}

Theorem II.1. For all nonzero elements $x_{1}, x_{2}, \ldots, x_{n}$ in a quasi-Banach space $\mathrm{X}$ with $\left\|x_{1}\right\| \geq\left\|x_{2}\right\| \geq \cdots \geq\left\|x_{n}\right\|$, then

$$
\begin{aligned}
\left\|\sum_{j=1}^{n} \frac{x_{j}}{\left\|x_{j}\right\|}\right\| & =\left\|\sum_{j=1}^{n} \frac{x_{j}}{\left\|x_{j}\right\|}+\sum_{j=1}^{n} \frac{x_{j}}{\left\|x_{i}\right\|}-\sum_{j=1}^{n} \frac{x_{j}}{\left\|x_{i}\right\|}\right\| \\
\leq & K\left\|\sum_{j=1}^{n} \frac{x_{j}}{\left\|x_{i}\right\|}\right\|+K^{2}\left\|\left(\frac{1}{\left\|x_{1}\right\|}-\frac{1}{\left\|x_{i}\right\|}\right) x_{j}\right\| \\
& +K^{2}\left\|\sum_{j=2}^{n}\left(\frac{1}{\left\|x_{j}\right\|}-\frac{1}{\left\|x_{i}\right\|}\right) x_{j}\right\|
\end{aligned}
$$

where $C$ is a constant and $C \geq 1$.

Proof: We follow the method of proof [8, Theorem 2.1], but make some essential modifications to it. First, let us see inequality (4): for a fixed $i \in\{1, \cdots, n\}$, we have

$$
\begin{aligned}
&\left\|\sum_{j=1}^{n} \frac{x_{j}}{\left\|x_{j}\right\|}\right\|=\left\|\sum_{j=1}^{n} \frac{x_{j}}{\left\|x_{j}\right\|}+\sum_{j=1}^{n} \frac{x_{j}}{\left\|x_{i}\right\|}-\sum_{j=1}^{n} \frac{x_{j}}{\left\|x_{i}\right\|}\right\| \\
& \leq K\left\|\sum_{j=1}^{n} \frac{x_{j}}{\left\|x_{i}\right\|}\right\|+K^{2}\left\|\left(\frac{1}{\left\|x_{1}\right\|}-\frac{1}{\left\|x_{i}\right\|}\right) x_{j}\right\| \\
&+K^{2}\left\|\sum_{j=2}^{n}\left(\frac{1}{\left\|x_{j}\right\|}-\frac{1}{\left\|x_{i}\right\|}\right) x_{j}\right\| .
\end{aligned}
$$

Hence, in order to prove inequality (4), let us set $C=\prod_{j=1}^{n} K_{j}$, where $K_{j}=K$ for all $1 \leqslant \mathrm{j} \leqslant \mathrm{n}$. Thus, 
from the above inequality it follows that

$$
\begin{aligned}
\left\|\sum_{j=1}^{n} \frac{x_{j}}{\left\|x_{j}\right\|}\right\| & \leq C\left\|\sum_{j=1}^{n} \frac{x_{j}}{\left\|x_{i}\right\|}\right\|+C \sum_{j=1}^{n}\left|\frac{1}{\left\|x_{i}\right\|}-\frac{1}{\left\|x_{j}\right\|}\right|\left\|x_{j}\right\| \\
& =\frac{C}{\left\|x_{i}\right\|}\left(\left\|\sum_{j=1}^{n} x_{j}\right\|+\sum_{j=1}^{n}\left|\left\|x_{j}\right\|-\left\|x_{i}\right\|\right|\right) .
\end{aligned}
$$

Let $x_{i}=x_{1}$. From the above inequality we get

$$
\begin{aligned}
\left\|\sum_{j=1}^{n} \frac{x_{j}}{\left\|x_{j}\right\|}\right\| & \leq \frac{C}{\left\|x_{1}\right\|}\left(\left\|\sum_{j=1}^{n} x_{j}\right\|+\sum_{j=1}^{n}\left(\left\|x_{1}\right\|-\left\|x_{j}\right\|\right)\right) \\
& \leq \frac{C}{\left\|x_{1}\right\|}\left\{\left\|\sum_{j=1}^{n} x_{j}\right\|+\left(n\left\|x_{1}\right\|-\sum_{j=1}^{n}\left\|x_{j}\right\|\right)\right\} .
\end{aligned}
$$

From this it follows that

$$
C \sum_{j=1}^{n}\left\|x_{j}\right\| \leq C\left\|\sum_{j=1}^{n} x_{j}\right\|+\left(n C-\left\|\sum_{j=1}^{n} \frac{x_{j}}{\left\|x_{j}\right\|}\right\|\right)\left\|x_{1}\right\|,
$$

which is inequality (4).

In order to prove inequality (3), we proceed in a similar way. For a fixed $i \in\{1, \cdots, n\}$, we have

$$
\begin{aligned}
\left\|\sum_{j=1}^{n} \frac{x_{j}}{\left\|x_{j}\right\|}\right\| & =\left\|\sum_{j=1}^{n} \frac{x_{j}}{\left\|x_{j}\right\|}+\sum_{j=1}^{n} \frac{x_{j}}{\left\|x_{i}\right\|}-\sum_{j=1}^{n} \frac{x_{j}}{\left\|x_{i}\right\|}\right\| \\
& =\left\|\sum_{j=1}^{n} \frac{x_{j}}{\left\|x_{i}\right\|}-\sum_{j=1}^{n}\left(\frac{1}{\left\|x_{i}\right\|}-\frac{1}{\left\|x_{j}\right\|}\right) x_{j}\right\| \\
& \geq \frac{1}{K}\left\|\sum_{j=1}^{n} \frac{x_{j}}{\left\|x_{i}\right\|}\right\|-\left\|\sum_{j=1}^{n}\left(\frac{1}{\left\|x_{i}\right\|}-\frac{1}{\left\|x_{j}\right\|}\right) x_{j}\right\| .
\end{aligned}
$$

From this it follows that

$$
\begin{aligned}
K\left\|\sum_{j=1}^{n} \frac{x_{j}}{\left\|x_{j}\right\|}\right\| & \geq \sum_{j=1}^{n} \frac{x_{j}}{\left\|x_{i}\right\|}\|-K\| \sum_{j=1}^{n}\left(\frac{1}{\left\|x_{i}\right\|}-\frac{1}{\left\|x_{j}\right\|}\right) x_{j} \| \\
\geq & \left\|\sum_{j=1}^{n} \frac{x_{j}}{\left\|x_{i}\right\|}\right\|-K^{2}\left\|\left(\frac{1}{\left\|x_{1}\right\|}-\frac{1}{\left\|x_{i}\right\|}\right) x_{j}\right\| \\
& -K^{2}\left\|\sum_{j=2}^{n}\left(\frac{1}{\left\|x_{j}\right\|}-\frac{1}{\left\|x_{i}\right\|}\right) x_{j}\right\| .
\end{aligned}
$$

Hence, in order to prove inequality (3), let us set $C=\prod_{j=1}^{n} K_{j}$, where $K_{j}=K$ for all $1 \leqslant \mathrm{j} \leqslant \mathrm{n}$. Thus, from the above inequality it follows that

$$
\begin{aligned}
C\left\|\sum_{j=1}^{n} \frac{x_{j}}{\left\|x_{j}\right\|}\right\| & \geq\left\|\sum_{j=1}^{n} \frac{x_{j}}{\left\|x_{i}\right\|}\right\|-C \sum_{j=1}^{n}\left|\frac{1}{\left\|x_{j}\right\|}-\frac{1}{\left\|x_{i}\right\|}\right|\left\|x_{j}\right\| \\
& =\frac{1}{\left\|x_{i}\right\|}\left\|\sum_{j=1}^{n} x_{j}\right\|-\frac{C}{\left\|x_{i}\right\|} \sum_{j=1}^{n} \mid\left\|x_{j}\right\|-\left\|x_{i}\right\| .
\end{aligned}
$$

Let $X_{i}=x_{n}$. From the above inequality we get

$$
\begin{aligned}
C\left\|\sum_{j=1}^{n} \frac{x_{j}}{\left\|x_{j}\right\|}\right\| & \geq \frac{1}{\left\|x_{n}\right\|}\left\|\sum_{j=1}^{n} x_{j}\right\|-\frac{C}{\left\|x_{n}\right\|} \sum_{j=1}^{n}\left(\left\|x_{j}\right\|-\left\|x_{n}\right\|\right) \\
& \geq \frac{1}{\left\|x_{n}\right\|}\left\|\sum_{j=1}^{n} x_{j}\right\|-\frac{C}{\left\|x_{n}\right\|} \sum_{j=1}^{n}\left\|x_{j}\right\|+n C .
\end{aligned}
$$

From this it follows that

$$
C\left\|\sum_{j=1}^{n} \frac{x_{j}}{\left\|x_{j}\right\|}\right\|\left\|x_{n}\right\| \geq\left\|\sum_{j=1}^{n} x_{j}\right\|-C \sum_{j=1}^{n}\left\|x_{j}\right\|+n C\left\|x_{n}\right\| .
$$

Therefore

$$
\left\|\sum_{j=1}^{n} x_{j}\right\|+C\left(n-\left\|\sum_{j=1}^{n} \frac{x_{j}}{\left\|x_{j}\right\|}\right\|\right)\left\|x_{n}\right\| \leq C \sum_{j=1}^{n}\left\|x_{j}\right\| .
$$

This completes the proof.

In fact, by the proof of Theorem (II.1) above, we can get the following results:

Theorem II.2. For all nonzero elements $x_{1}, X_{2}, \ldots, x_{n}$ in a quasi-Banach space $\mathrm{X}$

$$
\begin{aligned}
\| \sum_{j=1}^{n} x_{j} & +C\left(n-\left\|\sum_{j=1}^{n} \frac{x_{j}}{\left\|x_{j}\right\|}\right\|\right) \min _{1 \leq j \leq n}\left\{\left\|x_{j}\right\|\right\} \\
& \leq C \sum_{j=1}^{n}\left\|x_{j}\right\| \\
& \leq C\left\|\sum_{j=1}^{n} x_{j}\right\|+\left(n C-\left\|\sum_{j=1}^{n} \frac{x_{j}}{\left\|x_{j}\right\|}\right\|\right) \max _{1 \leq j \leq n}\left\{\left\|x_{j}\right\|\right\},
\end{aligned}
$$

where $C$ is a constant and $C \geq 1$.

Remark II.3. Notice that, when $\mathrm{n}=2$ in Theorem (II.1) we can get the inequalities (1) and (2) obtained by C. Wu and Y. J. Li in [1], which are evident from the results of our Theorem (II.1) and the proof of [1, Theorem 2.1](i.e., Theorem I.1 above)), here we omit it..

\section{ACKNOWLEDGMENT}

The author thanks to the referee for his (or her) helpful comments and suggestions. 


\section{REFERENCES}

[1] C. Wu and Y. J. Li, "On the triangle inequality in quasi-Banach spaces," J. Inequal. Pure Appl. Math. 9(2)(2008), Art. 41, 4. pp.

[2] M. Kato, K. S. Saito and T. Tamura, "Sharp triangle inequality and its reverse in Banach spaces," Math. Ineq. Appl. 10(2)(2007), 453462.

[3] K.-I. Mitai, K.-S. Saito, M.I. Kato and T. Tamura, "On sharp triangle inequalities in Banach spaces," J. Math. Anal. Appl. 336(2007), 1178-1186.

[4] S. S. Dragomir, "Reverses of the triangle inequality in Banach spaces," J. Inequal. Pure Appl. Math. 6(5)(2005), Art. 129, 46. pp.

[5] S. S. Dragomir, "Advances in inequalities of the Schwarz, triangle and Heisenberg type in inner product spaces," Nova Science Publishers, Inc. New York, 2007.

[6] F. Dadipour, M. S. Moslehian, J.M. Rassias and S.-E. Takahasi, "Characterization of a generalized triangle inequality in normed spaces," Nonlinear Anal-TMA. 75 (2012), no. 2, 735-741.

[7] Y. Benyamini and J. Lindenstrauss, "Geometric Nonlinear Functional Analysis," Vol. 1, Colloq. Publ. 48, Amer. Math. Soc. Providence, 2000.

[8] J. Pecaric and R. Rajic, "The Dunkl-Williams inequality with n elements in normed linear spaces," Math. Inequal. Appl. 10(2007), 461-470.

[9] M. Maligranda, "Some remarks on the triangle inequality for norms," Banach J. Math. Anal. 2 (2008), no. 2, 31-41.

[10] M. S. Moslehian, F. Dadipour, R. Raji c and R. Maric, "A glimpse at the Dunkl-Williams inequality," Banach J. Math Anal. 5 (2011), no. 2, 138-151. 